\title{
USO DE TERRITORIO E INTEGRACIÓN A LA ECONOMÍA DE MERCADO. ESTUDIO DE CASO EN LA AMAZONÍA BOLIVIANA
}

\author{
Cruz-Burga, Zoila. ${ }^{1}$ Reyes-García, Victoria. ${ }^{2}$ Alarcón Novoa, Jorge. ${ }^{3}$ \\ Paneque-Gálvez, Jaime. ${ }^{4}$ Luz, Ana C. ${ }^{4}$
}

Resumen

Abstract

Los pueblos indígenas de América Latina tienen problemas territoriales similares, en el sentido de que se enfrentan a intereses políticos y económicos que frenan la consolidación de sus derechos territoriales. Se alega a menudo que los grupos indígenas ya tienen suficientes tierras, que son relativamente pocos para tanto espacio, o que reclaman más de lo que son capaces de gestionar. El presente estudio tiene como objetivo analizar, desde una óptica geográfica y social el uso que los tsimane', un grupo indígena de cazadores-horticultores de la amazonia boliviana, hacen de su territorio. El análisis se enfoca en señalar las diferencias de usos del territorio en función del nivel de integración a la economía de mercado de las diferentes comunidades. Se utilizó información geográfica obtenida en el periodo 2008-2009 mediante un proyecto de mapeo participativo en parte del territorio habitado por los tsimane', así como información socioeconómica procedente de encuestas individuales llevadas a cabo en 32 comunidades tsimane'. Los resultados obtenidos evidencian que la densidad poblacional, la ganancia de ingresos y la cercanía a carreteras y caminos forestales están directamente asociados a la superficie utilizada por la comunidad; mientras que el grado de actividad comercial, desarrollada por la comunidad y la cercanía de esta al mercado, están inversamente relacionados con el área total aprovechada. Nuestro estudio remarca la importancia del conocimiento de las áreas utilizadas y de cómo se relacionan con sus actividades económicas y subsistencia en la planificación y ordenamiento del territorio indígena.

Palabras clave: Tsimane', Bolivia, Amazonía, territorios indígenas, SIG.
Indigenous people in Latin America have similar territorial problems, facing political and economic interests that hamper the consolidation of their territorial rights. It is often alleged that indigenous groups already have enough land, that they are relatively few for so much territory, or that they claim more land than they are able to manage. The present study aims to analyze, from a geographical and social perspective, how the Tsimané, a hunter-horticulturalist indigenous group from the Bolivian Amazon, use their territory. The study focuses on potential differences in territory use by the various communities, according to their level of integration into the market economy. We used geographic information obtained in 20082009 by means of a participatory mapping project carried out in part of the territory inhabited by the Tsimané, as well as socioeconomic data from individual surveys conducted in 32 Tsimané villages. Results suggest that population density, income and proximity to highways and forest roads are directly associated with the total area used by the community. On the contrary, commercial activity and closeness to the market are inversely related to the total area used. Our study highlights the importance of knowing the areas used by Amazonian indigenous peoples, and how they relate to their subsistence and economic activities for territorial planning and management.

Key words: Tsimane', Bolivia, Amazon, indigenous territories, GIS. 


\section{Introducción}

Desde la colonización europea de América, los pueblos indígenas han sufrido y reclamado por la continua pérdida de acceso a sus tierras y recursos naturales. Cada región y cada pueblo indígena tiene su historia particular de estos ciclos de conflicto y protesta (Smith, 2003). A partir de la década de 1980, los países de América Latina empezaron a incorporar en sus constituciones disposiciones sobre derechos de los pueblos indígenas, especialmente en relación con la tierra y el territorio (OIT, 2009). Estos avances en política no significan necesariamente que las disposiciones acordadas sean puestas en práctica, pues como señalan algunos autores (Stocks, 2005), no es fácil cambiar más de 500 años de colonización y postcolonización cuando el tema involucra algo tan esencial como la tierra.

En el debate sobre los derechos de los pueblos indígenas sobre el territorio existen dos posiciones contrarias. Por un lado, están los que argumentan que los territorios indígenas son tierras despobladas y no productivas. De acuerdo con esta corriente, algunos políticos, empresarios e investigadores ven en los territorios indígenas oportunidades de inversión y están a favor de la titulación individual de los territorios y la creación de empresas, las cuales ayudarían a controlar el territorio, proteger los valores culturales y vencer la pobreza (García, 2007; De Soto, 2009, 2010). La otra corriente de pensamiento sostiene que el uso indígena del territorio es de tipo extensivo (i.e., sustentable) y por ello ayuda a la conservación de la biodiversidad, a la vez que promueve el derecho de los indígenas sobre el territorio que tradicionalmente han ocupado. En esta corriente se encuentra un amplio grupo de investigadores que afirma que los pueblos indígenas, al vivir en armonía con su entorno durante milenios, han adquirido un conocimiento ecológico tradicional que les permite conservar o usar de manera más sostenible esos territorios y sus recursos naturales (Toledo, 2003; Sobrevila, 2008).

No obstante, los proponentes de esta visión también argumentan que el proceso de integración a la economía de mercado, así como el aumento poblacional y la adquisición de tecnologías más modernas están haciendo que algunas sociedades indígenas tengan usos más extractivos y destructivos del medio ambiente (Redfort, 1991; Rudel et al., 2002; Vadez et al., 2004; Godoy et al., 2005).

Estas dos visiones sobre los derechos de los indígenas sobre el territorio han generado políticas nacionales diferentes. Así, la primera ha promovido políticas que priorizan la ocupación y utilización de los territorios para fines productivos (e.g., hidrocarburos, minería, centrales hidroeléctricas, concesiones forestales, agricultura comercial a gran escala), frecuentemente bajo una lógica neoliberal (Pacheco, 2006; De Soto, 2009). La segunda visión ha llevado a que diversas instituciones apoyen a los pueblos indígenas en la defensa de los derechos sobre sus tierras (Chapín et al., 2005; (Assies, 2006) Reyes-García et al., 2010).

A pesar de la importancia que para los pueblos indígenas tiene el uso de sus territorios y de los recursos que en ellos se encuentran, hasta el momento existen pocos estudios que analicen el uso que los indígenas hacen de su espacio con una óptica geográfica ${ }^{1}$, y menos aún que relacionen el uso del espacio con variables socioeconómicas. En este trabajo se aborda este tema analizando el uso que hace la población tsimane' de su territorio. Específicamente, se analiza el aprovechamiento de recursos silvestres como caza, pesca, extracción de productos forestales

106 'Se refiere a la consideración espacial y georeferenciada de las diferentes actividades cotidianas de los Tsimane'. 
maderables (PFM) y productos forestales no maderables (PFNM), determinando hasta qué punto la superficie utilizada por las comunidades está relacionada con el nivel de integración a la economía de mercado. De esta forma, se desea saber si las comunidades con mayores ingresos económicos, mayor actividad comercial, mayor número de hogares, y más cercanas al mercado utilizan territorios más extensos. También se desea comprobar si la presencia de carreteras o caminos forestales está asociada a la superficie usada por la comunidad.

Entendemos que una mejor comprensión de la relación entre superficie territorial utilizada y las actividades económicas y de subsistencia permitirá un mejor ordenamiento del territorio tsimane'. Asimismo, permitirá la mejora de estrategias de planificación y desarrollo en un escenario en el que la necesidad de espacio por las comunidades indígenas y la intensidad de aprovechamiento sobre los bosques puede ir en aumento.

\section{Los Tsimane' y su territorio}

El pueblo tsimane' (o chimane) está confor- mado por unas 15.000 personas agrupadas en aproximadamente 125 comunidades, la mayoría ubicada en el departamento de Beni, Bolivia. Tradicionalmente, los tsimane' habitaban en las proximidades de los ríos Maniqui, Quiquibey y Sécure, pero en la actualidad se distribuyen también a lo largo del eje carretero Yucumo-Rurrenabaque y a lo largo de pistas madereras en concesiones forestales (Reyes-García et al., 2012a). Asimismo, las comunidades tsimane' se ubican en la actualidad en áreas con diferente tipo de tenencia, incluyendo tierras indígenas ${ }^{2}$, áreas protegidas, concesiones forestales y tierras privadas (Guèze, 2011).

El territorio indígena tsimane' $(\mathrm{TICH})$ fue reconocido como tierra comunal en 1990; en 1996 fue establecida como tierra comunitaria de origen (TCO) ${ }^{3} y$ finalmente, en 2010, pasó a llamarse territorio indígena originario campesino $(\mathrm{TIOC})^{4}$. EI TICH concentra la mayor parte de la población tsimane' y el 70\% de su superficie pertenece al municipio de San Borja, en el Beni (Melgar, 2009). El TICH abarca poco menos de 400.000 hectáreas, y se extiende desde la base de los Andes hacia los bosques tropicales del noreste de Bolivia (Figura 1).

Figura 1. Comunidades tsimane' objeto de estudio incluyendo los regímenes de tenencia de la tierra en los que se ubican

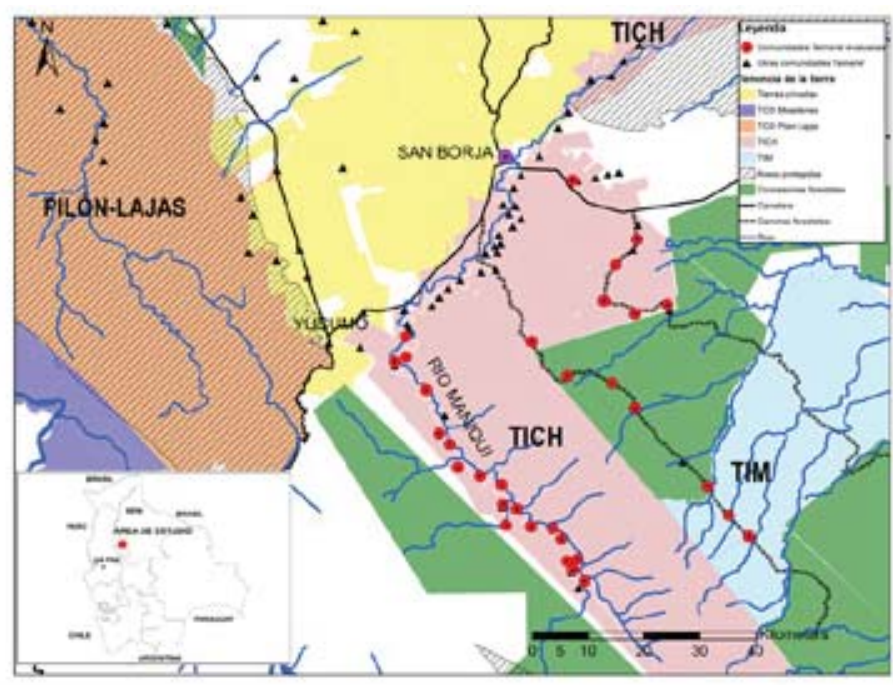

2 Tierras comunitarias de origen (TCO) presentes en esta zona de la Amazonía boliviana: territorio indígena chimane (TICH), territorio indígena multiétnico del bosque de Chimane (TIM ), territorio indígena y parque nacional Isiboro Sécure (TIPNIS) y Pilón Lajas (Melgar, 2009). 
Los tsimane' varían con relación a su integración a la economía de mercado: algunos tsimane' son monolingües en lengua tsimane' y viven en comunidades prácticamente autárquicas, alejados del mercado (ciudades de San Borja, Yucumo, etc.). Otros tsimane' también hablan bien el español y viven en comunidades con gran influencia del mercado, en asentamientos más grandes y accesibles por carretera (Reyes-García et al., 2010). A pesar de estas diferencias, en mayor o menor medida todos los tsimane' siguen dependiendo de los recursos del bosque para cazar, pescar y recolectar productos. La agricultura de subsistencia continúa siendo la principal actividad económica de los tsimane' (Reyes-García, 2001; Vadez et al., 2008).

Uso del bosque y recursos naturales El derecho de los tsimane' al uso del territorio y los recursos naturales depende del tipo de tenencia de la tierra en el que viven. Las comunidades asentadas en las TCO tienen derecho a abrir áreas de bosque para hacer cultivos, así como a cazar, pescar, y extraer PFM y PFNM para su consumo. Si el aprovechamiento forestal es con fines comerciales, han de tener aprobado un plan de manejo y es el Gran Consejo Tsimane' -el órgano representativo político de los tsimane' - quien lo aprueba. Las comunidades asentadas en áreas protegidas y en concesiones forestales pueden realizar actividades de subsistencia pero no pueden extraer PFM y PFNM sin permisos ${ }^{5}$. Por último, las pocas comunidades que se ubican en tierras privadas (haciendas) no tienen derecho a utilizar los recursos naturales y en estas áreas los indígenas suelen trabajar para los hacendados (Guèze, 2011).

Actividades de subsistencia La agricultura tsimane' se caracteriza por el uso de la roza-tumba-quema. Los cultivos principales son el arroz, la yuca, el maíz, y el plátano. Tradicionalmente, los tsimane' utilizan sus parcelas de cultivo un máximo de tres años, tras los cuales dejan el terreno en barbecho para ser reutilizado entre 5 y 30 años después (Huanca, 1999; Vadez et al., 2008). Mientras tanto, los barbechos son una fuente de leña, maderas para utensilios e importante lugar de caza de roedores.

El consumo de los recursos naturales cubre gran parte de las necesidades de subsistencia de los tsimane', además de ser una fuente de ingreso monetario. Se ha determinado que el $52,5 \%$ de los productos consumidos por las comunidades proviene del bosque y, de este porcentaje, el 36\% corresponde al consumo de proteína animal. Así, un hogar tsimane' típico puede consumir hasta unos 200 kilos de pescado y 150 kilos de carne silvestre al año (Reyes-García et al., 2002).

Respecto al aprovechamiento de la fauna silvestre, Apaza (2002) determinó que una comunidad alejada del mercado local utilizaba un área de caza de 16.424 ha en comparación con un área más cercana que utilizaba 1.945 ha. En otros estudios sobre la caza en otras comunidades se determinó que el área de uso puede variar desde 3.070 ha a 19.550 ha (Chicchón, 1994; Gutiérrez, 2005).

\section{Actividades comerciales}

Aunque los tsimane' practican mayoritariamente una agricultura de subsistencia, las poblaciones de la parte central y la parte baja del río Maniqui se caracterizan por comercializar algunos productos agrícolas como el arroz, el plátano, y otros productos (Lieberman, 2000; Pérez, 2001). La agricultura comercial está orientada principalmente a la venta de arroz. De acuerdo a los últimos estudios, el área bajo este cultivo ha aumentado significativamente en los últimos años, lo cual ha producido mayor deforestación 
en el área (Vadez et al., 2008). En promedio, cada hogar abre al año 0,27 ha de bosque para cultivar arroz, de las cuales 0,12 ha son en bosque maduro y 0,15 ha de bosque secundario (Vadez et. al., 2008).

Las comunidades tsimane' ubicadas en la parte superior del río Maniqui basan buena parte de su economía en la venta de paños elaborados a partir de la hoja de una palma localmente conocida como jatata (Geonoma deversa) ${ }^{6}$. Esta comercialización se realiza de forma directa en la ciudad de San Borja o a través de la venta o trueque con comerciantes que visitan las comunidades tsimane' con productos que traen de la ciudad. En la actualidad y debido a la alta comercialización de este producto existen varias ONG e instituciones que trabajan para promover su manejo y comercialización de forma sustentable.

La mara o caoba (Swietenia macrophylla) es el PFM más importante y conflictivo en la zona, principalmente en el TIM, donde los conflictos entre empresarios madereros, extractores ilegales e indígenas se han sucedido durante décadas a pesar de los varios intentos de desarrollar propuestas de manejo integral del bosque. Otras maderas comerciales en la zona son el cedro (Cedrela spp.), el palo maría (Calophyllum brasiliense), el roble (Amburana cearensis), el almendrillo (Pouteria spp.), el verdolago (Terminalia spp.), y el ochoó (Hura crepitans). Los tsimane' frecuentemente trabajan como extractores asalariados de madera o jatata para las empresas madereras o para comerciantes ilegales que entran en sus territorios.

\section{Conflictos por el acceso a los recursos y territorio} Actualmente los principales problemas que enfrentan los Tsimane' son el reconocimiento de sus tierras ancestrales y el acceso a los recursos naturales en estas tierras. Si bien en la última década los cambios legislativos y de constitución han jugado un papel importante en la legitimización de los derechos de los pueblos indígenas y sus territorios, en la práctica sigue habiendo amenazas que deben ser enfrentadas. Actualmente, una de las mayores amenazas para el territorio tsimane' es la posibilidad de arribo a la zona de un mayor número de migrantes colonos que pudieran llegar como consecuencia de la nueva política gubernamental de distribución de tierras. Otros conflictos por el territorio también podrían deberse al mejoramiento y construcción de caminos y carreteras, y al desarrollo de industrias extractivas como hidrocarburos y minería (Reyes-García et al., 2012a; 2012b).

\section{Métodos}

El estudio evalúa 32 comunidades tsimane', de las cuales 19 se encuentran a lo largo del río Maniqui y 13 cerca de caminos secundarios o en la carretera principal que va de Yucumo a San Borja (ver gráfico $N^{\circ} 1$ ). Con respecto a la tenencia de tierras, 26 comunidades están en el TICH, dos en el TIM y cuatro en la concesión forestal de la empresa Fátima. La cobertura vegetal predominante es de bosques densos verdes lluviosos, tanto no inundables (i.e., terra firme) como inundables, con uso principalmente forestal y una pequeña parte, hacia el norte, cerca de San Borja, Manguito y Tacuaral del Mato, de uso silvo-pastoril (Superintendencia Agraria, 2001).

Mapeo participativo

El mapeo participativo se realizó durante los años 2008 y 2009. La conducción del proyecto y el levantamiento de información de campo fue hecha por tres investigadores de la Universidad Autónoma de Barcelona (UAB, Barcelona, España) y cuatro técnicos

${ }^{4}$ De acuerdo al Decreto Supremo No727 (2010) las TCO pasan a denominarse territorio indígena originario campesino (TIOC). Un TIOC se define como el territorio ancestral sobre el cual se constituyeron las tierras colectivas o comunitarias de origen debidamente consolidadas conforme a ley y que ha adquirido esta categoría mediante el procedimiento administrativo ante la autoridad agraria. Un TIOC podrá dar paso a la conformación de un gobierno autónomo indígena originario campesino (Ley Marco de Autonomías y Descentralización Andrés Ibáñez, Ley Nº31), el cual deberá ser aprobado por ley y será reconocido como una unidad territorial. 
tsimane'. Se realizaron dos visitas a cada una de las 32 comunidades con el fin de 1) realizar un taller para dar a conocer el proyecto a los comuneros, así como las ventajas y desventajas del mapeo participativo; 2) realizar mapas participativos en los cuales los comuneros caracterizaban el territorio y el uso que la comunidad hacía del mismo; y 3) realizar recorridos en campo para tomar las coordenadas geográficas de los lugares dibujados en el mapa con el Sistema de Posicionamiento Global (GPS). Los puntos georeferenciados incluyeron 1) fisiografía (e.g., ríos, lagos) e infraestructura de importancia (e.g., caminos, casas); 2) áreas con usos determinados como campamentos de caza y de pesca, áreas de extracción de madera, parches de palmeras, árboles frutales o especies medicinales; y 3) sitios de importancia cultural e histórica como lugares sagrados y sitios arqueológicos. La descripción detallada de la metodología seguida en este trabajo se encuentra en Reyes-García et al., (2012b).

\section{Entrevistas}

La información socioeconómica proviene de entrevistas realizadas durante el mapeo participativo. En cada una de las 32 comunidades se realizaron entrevistas estructuradas con un dirigente y se recogió información que permitió caracterizar a las comunidades de estudio (total de hogares en la comunidad, principales actividades económicas, etc.). Además, en cada comunidad se realizaron de 3 a 27 entrevistas, dependiendo del número total de familias en la comunidad, seleccionadas aleatoriamente a partir de una lista de hogares dada por el máximo dirigente de la comunidad. Se entrevistó únicamente al jefe de familia para recoger información a nivel de hogar (i.e., número de integrantes, grado de educación, ingresos por venta de productos, principales actividades de sub- sistencia, etc.). Se recogió, en total, información de 360 hogares.

\section{Análisis de datos}

El análisis de datos se desarrolló en dos partes. Primero, para caracterizar el uso actual del territorio se determinó el área de uso frecuente por comunidad y la distancia promedio recorrida por los pobladores de cada comunidad en sus actividades de caza, pesca, extracción de PFM y PFNM. Segundo, para analizar los factores o variables que tienen mayor influencia en la extensión del área de uso frecuente o en las distancias recorridas; se planteó y utilizó un modelo de regresión multivariado.

\section{Determinación del espacio utilizado} Para hallar las áreas utilizadas en las diferentes actividades y las distancias de aprovechamiento de las comunidades analizadas se utilizaron 7.869 puntos georeferenciados procedentes de la base de datos del mapeo participativo. A partir de estos puntos se determinó para cada comunidad: 1) el área total utilizada, 2) las áreas utilizadas para diferentes actividades de subsistencia, y 3) las distancias recorridas para aprovechar los recursos.

Las áreas totales de uso por comunidad se calcularon con ARCGIS 9.3 utilizando el método de envolventes convexos, el cual solo considera los puntos externos de un conjunto de puntos para delimitar un polígono o área (ver gráfico $N^{\circ} 2$ ). En la determinación de las áreas de aprovechamiento total se incluyeron los puntos con información relativa a todos los usos de cada comunidad. Para la determinación de las áreas destinadas a las diferentes actividades de subsistencia (caza, pesca, extracción de madera, y extracción de jatata), se siguió la misma metodología pero tomando en

$110{ }^{5}$ Sus actividades deben estar en conformidad con las normas establecidas en los planes de manejo de estas áreas (Reglamento de Áreas Protegidas Decreto Supremo N²4781; Ley Forestal Ley N¹700; Ley del Servicio Nacional de Reforma Agraria Ley $\left.N^{\circ} 1715\right)$. 
cuenta únicamente los puntos referidos a cada actividad. Adicionalmente, para estimar las distancias de aprovechamiento de los recursos de cada comunidad se utilizó la extensión Network Analyst de ARCGIS 9.3. Esta extensión permitió generar rutas eficientes entre los puntos de extracción utilizando los recorridos habituales de los pobladores y determinar la distancia más próxima entre sitios de importancia (i.e., centro de la comunidad - punto de extracción del recurso). La distancia entre cada comunidad y la ciudad de San Borja fue estimada también con esta metodología teniendo en cuenta las rutas usualmente utilizadas por los pobladores.

\subsection{Especificación del modelo estadístico}

Debido a que la unidad de observación y análisis es la comunidad, se agregaron las respuestas de las entrevistas de hogar con el fin de generar variables promedio en el nivel de comunidad (32 comunidades). La variable dependiente en nuestro modelo es el área de aprovechamiento total utilizada por cada comunidad, y las variables explicativas utilizadas fueron distancia al mercado principal (San Borja), número de hogares, promedio de ingreso por hogar, ubicación de la comunidad con respecto al río Maniquí, y principales actividades económicas de la comunidad (ver cuadro $N^{\circ} 1$ ). La distancia al mercado fue estimada a partir de las rutas

Cuadro 1. Descripción de variables incluidas en el modelo estadístico

\begin{tabular}{|c|c|c|}
\hline Variables & Descripción & Fuente de Información \\
\hline \multicolumn{3}{|l|}{ Dependiente } \\
\hline Área total & $\begin{array}{l}\text { Superficie utilizada por la co- } \\
\text { munidad para el desarrollo de } \\
\text { sus actividades. Incluye áreas } \\
\text { de caza, pesca, recolección de } \\
\text { productos forestales maderables } \\
\text { y no maderables en hectáreas. }\end{array}$ & $\begin{array}{l}\text { Determinada con ArcGis } 9.3 \text { a } \\
\text { partir de los puntos tomados con } \\
\text { GPS de la base de datos del pro- } \\
\text { yecto Mapeo Participativo } 2008- \\
2009 \text { (BDPMP) }(n=32) \text {. }\end{array}$ \\
\hline \multicolumn{3}{|l|}{ Explicativas } \\
\hline Distancia al mercado & $\begin{array}{l}\text { Kilómetros desde la comunidad } \\
\text { a la ciudad de San Borja vía río, } \\
\text { carretera o camino secundario. }\end{array}$ & $\begin{array}{l}\text { Determinada con ArcGis } 9.3 \text { a } \\
\text { partir de los puntos tomados con } \\
\text { GPS de la BDPMP }(n=32) \text {. }\end{array}$ \\
\hline Hogares & $\begin{array}{l}\text { Número de hogares en la comu- } \\
\text { nidad. }\end{array}$ & $\begin{array}{l}\text { Obtenida a partir de la BDPMP y } \\
\text { entrevistas a nivel de comunidad } \\
(n=32) \text {. }\end{array}$ \\
\hline Ingreso hogar & $\begin{array}{l}\text { Ingreso promedio de un hogar } \\
\text { de la comunidad en los últimos } \\
\text { tres meses antes de la entrevista. } \\
\text { Incluye ventas de arroz y jatata, y } \\
\text { trabajo asalariado. } \\
\text { (1 US } \$=7.00 \mathrm{Bs} \text { ) }\end{array}$ & $\begin{array}{l}\text { Obtenida de la BDPMP. Informa- } \\
\text { ción que proviene de entrevistas } \\
\text { en los hogares ( } n=360 \text { ) agrega- } \\
\text { das en el nivel de comunidad } \\
(n=32) \text {. }\end{array}$ \\
\hline Influencia del río & $\begin{array}{l}\text { Ubicación de la comunidad con } \\
\text { respecto a la cercanía del río } \\
\text { Maniqui; se consideró que las } \\
\text { comunidades cuyo centro está } \\
\text { a menos de } 2 \mathrm{~km} \text { del río tienen } \\
\text { influencia ( } 0=\sin \text { influencia; } 1=\text { con } \\
\text { influencia). }\end{array}$ & $\begin{array}{l}\text { Determinada con ArcGis } 9.3 \text { a } \\
\text { partir de los puntos tomados con } \\
\text { GPS de la BDPMP. }\end{array}$ \\
\hline Grado de actividad comercial & $\begin{array}{l}\text { Presencia de actividades eco- } \\
\text { nómicas comerciales. Se de- } \\
\text { finió utilizando las principales } \\
\text { actividades económicas de la } \\
\text { comunidad, considerándose a la } \\
\text { agricultura de subsistencia, caza } \\
\text { y recolección como actividades } \\
\text { no comerciales y a la agricultura } \\
\text { comercial, ganadería, venta de } \\
\text { madera y jatata como actividades } \\
\text { comerciales ( }(0=\text { no comercial, } \\
1=\text { comercial). }\end{array}$ & $\begin{array}{l}\text { Obtenida de BDPMP y entrevis- } \\
\text { tas a nivel de comunidad }(n=32) \text {. }\end{array}$ \\
\hline
\end{tabular}


usualmente utilizadas por los pobladores para llegar desde el centro de su comunidad. La variable ingreso económico registra el ingreso promedio de un hogar en la comunidad en tres meses por ventas de arroz, jatata y trabajo asalariado. El grado de actividad comercial se estimó a partir de las entrevistas a 32 dirigentes de comunidad y mide las dos principales actividades económicas predominantes en cada comunidad. Se consideraron como actividades no comerciales la agricultura de subsistencia, la caza y la recolección, mientras que la venta de madera y jatata se consideraron como actividades comerciales. Para estimar la asociación de estas variables sobre el área utilizada por las comunidades se desarrolló la siguiente relación funcional implícita:

$$
Y_{i}=f\left[X_{1 i}, X_{2 i}, X_{3 i}, D_{1}, D_{2}, \mu_{i}\right]
$$

Donde

$Y_{i}$ es la superficie total utilizada por la comunidad i.

$\mathrm{X}_{11}$ es la distancia de la comunidad $i$ a la ciudad de San Borja.

$X_{2 i}$ es el número de hogares en la comunidadi.

$\mathrm{X}_{3 \mathrm{i}}$ es el ingreso promedio de un hogar en la comunidad i.

$D_{1 i}$ es una variable dicotómica que permite evaluar las diferencias entre comunidades cercanas al río Maniqui versus comunidades no cercanas al río. ( $0=\sin$ influencia, $1=$ con influencia).

$\mathrm{D}_{2 i}$ es una variable dicotómica que evalúa diferencias entre comunidades con actividad comercial versus las que tiene poca o nula actividad comercial $(0=$ no comercial, $1=$ comercial).
La estimación del modelo se realizó con el software E-Views 7.0. Después de varias especificaciones funcionales (entre las que destacan la lineal, semi-logarítmica y doblelogarítmica), así como el uso de algunos indicadores de la bondad de ajuste (coeficientes de determinación, error estándar de la regresión, prueba F múltiple y los coeficientes Akaike y Schwarz), se determinó que la ecuación funcional que mejor representa el ajuste a los datos disponible fue la función doble-logarítmica siguiente:

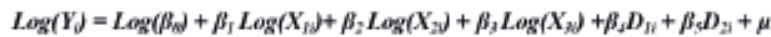

\section{Resultados}

La ciudad de San Borja se encuentra a una distancia promedio de las comunidades evaluadas de $53 \mathrm{~km}$ lineales o $98 \mathrm{~km}$ siguiendo el recorrido por río o caminos. Las comunidades asentadas en el río Maniqui están ubicadas a una distancia media de $3,8 \mathrm{~km}$ $(\mathrm{DS}=1,7)$ una de otra, mientras que las ubicadas en caminos están a un promedio de $6,5 \mathrm{~km}(\mathrm{DS}=1,1)$ una de otra. El número de hogares por comunidad varía de 3 a 95, con una media de 25 hogares. El número de integrantes por hogar es en promedio seis personas y el ingreso trimestral promedio familiar proveniente de venta de arroz, jatata y trabajo asalariado es de 858 bolivianos o 122,57 dólares americanos (ver cuadro $N^{\circ} 2$ ). 
Cuadro 2. Estadística descriptiva de las variables analizadas ( $n=32$ comunidades)

$\begin{array}{lcccr}\text { Variables } & \text { Prom. } & \text { DS } & \text { Min. } & \text { Max. } \\ & & & & 26.474,11 \\ \text { Área total (ha) } & 8.862,96 & 5.939,37 & 1.387,66 & 23.979,36 \\ \text { Área de caza (ha) } & 5.734,97 & 4.899,01 & 44,66 & 18.366,86 \\ \text { Área de pesca (ha) } & 5.324,31 & 4.299,00 & 33,46 & 4.762,41 \\ \text { Área de extracción de } & 1.168,94 & 1.233,03 & 0,00 & 25.262,96 \\ \text { jatata (ha) } & & & & \\ \text { Área de extracción de } & 6.644,63 & 5.303,35 & 421,22 & 19,76 \\ \text { madera (ha) } & & & & 9,76 \\ \text { Distancia de caza (km) } & 6,42 & 3,33 & 1,32 & 18,51 \\ \text { Distancia de pesca (km) } & 5,52 & 2,09 & 1,83 & 13,30 \\ \text { Distancia de extracción de } & 6,63 & 2,98 & 3,21 & 174,72 \\ \text { jatata (km) } & & & 1,58 & 95,00 \\ \text { Distancia de extracción de } & 5,83 & 2,55 & 14,96 & 9,00 \\ \text { madera (km) } & & & 3,00 & 3.032,08 \\ \text { Distancia al mercado (km) } & 98,28 & 46,06 & 4,00 & 1.280,00 \\ \text { Número de hogares } & 24,50 & 22,35 & 110,00 & 282,31 \\ \text { Personas por familia } & 6,02 & 1,02 & 46,26 & \\ \text { Ingreso trimestral (Bs) } & 857,88 & 559,73 & 6,14 & \\ \text { Relación área/hogar } & 469,72 & 302,10 & 59,70 & \\ \text { Relación área/persona } & 82,97 & & & \end{array}$

\section{Espacio utilizado y distancias recorridas}

En promedio, las comunidades tsimane' evaluadas utilizan un área aproximada de 8.863 ha $(469,7$ ha/hogar o 82,4 ha/persona) en el desarrollo de sus actividades de subsistencia (caza, pesca, extracción de PFM y PFNM) (ver gráfico $\mathrm{N}^{\circ}$ 2). Es importante resaltar que, por lo general, las áreas de aprovechamiento determinadas en cada comunidad son de uso múltiple, es decir, que el mismo espacio es utilizado para la extracción de diferentes recursos y que entre comunidades cercanas se observan áreas superpuestas en las zonas
En términos generales los tsimane' utilizan una mayor extensión para la extracción de madera que para otras actividades productivas, siendo esta en promedio de 6.645 ha. El área promedio de caza es de 5.735 ha y de aprovechamiento de jatata es 1.289 ha. En cuanto a los desplazamientos por los caminos, los tsimane' recorren en promedio distancias que van de 1,8 a 9,8 km para la pesca, 1,6 a 13,3 km para la extracción de madera, de 1,3 a 19,8 km para la caza y de 3,2 a $18,5 \mathrm{~km}$ para la extracción de jatata (ver cuadro $\mathrm{N}^{\circ} 2$ y gráfico $\mathrm{N}^{\circ} 3$ ). 
Figura 2. Áreas de aprovechamiento total por comunidad

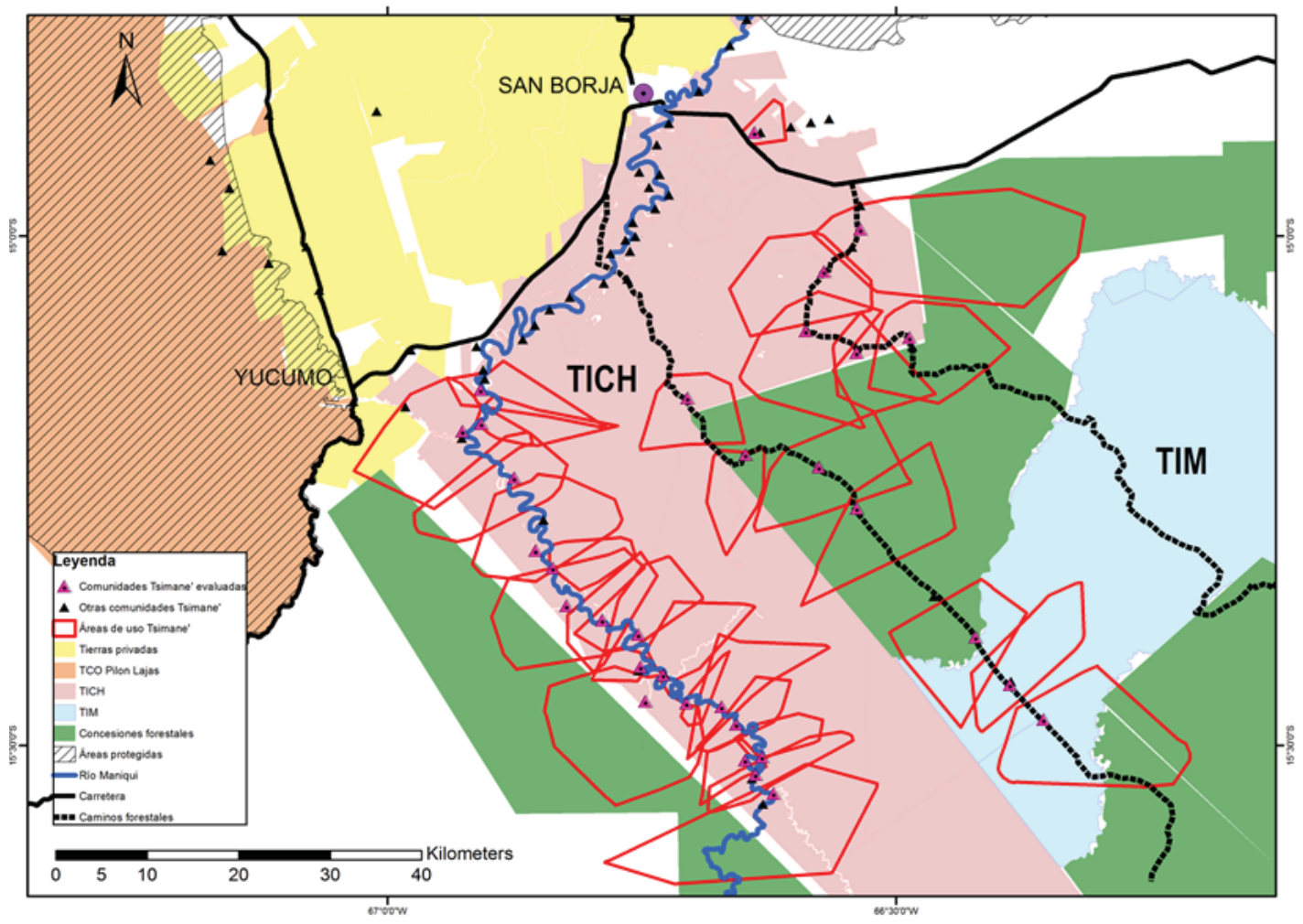

Resultados de la estimación del modelo estadístico

En la aplicación del modelo de regresión multivariado, cuatro de las cinco variables propuestas (distancia al mercado, número de hogares, grado de actividad comercial, e influencia del río Maniqui) están asociadas significativamente (5\% de margen de error) con el área total utilizada por comunidad. El ingreso monetario utilizado también tiene una asociación positiva con el área total utilizada por la comunidad, pero con un margen de error del 10\%. Los resultados presentados en el cuadro $\mathrm{N}^{\circ} 3$ fueron obtenidos mediante el método de mínimos cuadrados ordinarios. 
Figura 3. Promedios de distancia recorrida por comunidad y por recurso.

De mayor a menor

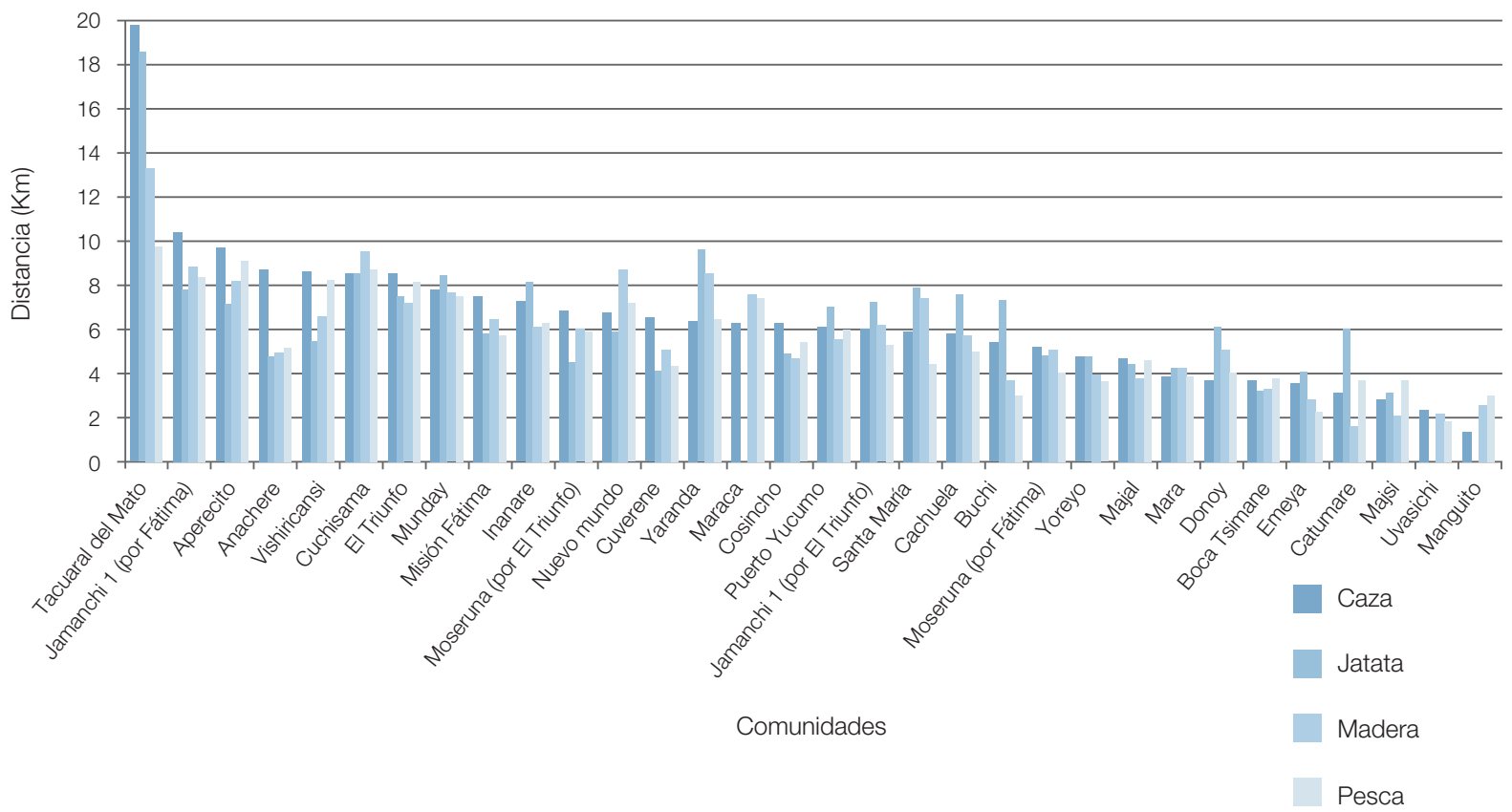

Cuadro 3. Resultados de modelo de regresión doble logarítmica

\section{Variable}

C (intercepto)

Distancia al mercado, en logaritmos

Número de hogares, en logaritmos

Ingreso hogar, en logaritmos Influencia del río

Grado de actividad comercial

$\mathrm{R} 2$

R2 ajustado

Error estándar de la regresión 0,3958

Estadístico $\mathrm{F}$

Prob (Estadístico F)

\section{Coeficiente Error Estándar t-Estadístico}

Prob.

0,7174
1,1812

0,9129

0,2149

$-1,5518$

$-0,3451$

0,7833

0,7416

Media de la variable dependiente

Desv. estándar de la variable dependiente

18,7935

0,00000
8,8337

0,7787

0,6521

0,0001

0,0000

0,0822

0,0000

0,0371

Estad. Durbin-Watson

2,0312 
De esta forma, los coeficientes parciales del modelo doble logarítmico indican que aquellas comunidades más distantes de la ciudad de San Borja aprovechan mayor superficie de bosques (con una elasticidad de 1,18\%). Tomando como referencia la distancia y superficie promedio del área en estudio, un aumento en la distancia de $1 \mathrm{~km}$ a San Borja estaría asociado a un aumento en el área utilizada de 104,5 ha. De forma similar, si el número de hogares (por comunidad) fuera $1 \%$ mayor, en promedio, el área bajo uso se incrementaría 0,91\%, lo que significaría que incrementar la presión familiar en promedio de 1 familia por comunidad estaría asociado a un aumento de 322,6 ha en el área utilizada (aproximadamente el 68\% del uso actual por familia). Asimismo, manteniendo las otras variables constantes, un incremento del ingreso en 1\% está asociado con un incremento del área utilizada en 0,2\% (aunque un incremento en el ingreso no necesariamente se asocia con un mayor vínculo al mercado).

Por otro lado, los resultados de la regresión con las variables dicotómicas indican que las comunidades que tienen mayor dedicación a actividades comerciales, en promedio, utilizan menos de la mitad del área que las comunidades que se dedican básicamentea actividades de subsistencia. Nuestros resultados también muestran que existe una asociación negativa entre la variable influencia del río Maniqui y el área utilizada. Así, las comunidades cercanas al río utilizan en promedio 34,5\% menos territorio que aquellas comunidades más influenciadas por caminos secundarios o carreteras.

En análisis no mostrado, también probamos la asociación entre área total usada y 1) ubicación de la comunidad (dentro o fuera de la TCO tsimane'), 2) la cantidad de bienes de mercado como escopetas y motosierras, y 3 ) el número promedio de viajes realizados por los pobladores a la ciudad de San Borja. Nin- guna de estas variables resultó estar significativamente asociada a la superficie utilizada por una comunidad y debido al pequeño tamaño de muestra ( $n=32)$, omitimos estas variables del modelo final, atendido al principio de "parsimonia" para los modelos de regresión múltiple, es decir para no perder grados de libertad (Gujarati y Porter, 2010, pp. 42).

\section{Discusión}

En el presente estudio centramos la discusión de nuestros resultados en tres temas. Primero, la sobreposición de usos y usuarios en el territorio usado por los tsimane'; segundo, la extensión e intensidad de los usos del territorio, y por último, las implicaciones de nuestros resultados en la planificación del territorio.

Sobre posición de usos y usuarios En la determinación de las superficies de aprovechamiento de las 32 comunidades se observa claramente que existen varias áreas que son aprovechadas para diferentes fines y que a su vez son utilizadas por diferentes comunidades. Este aprovechamiento intensivo en determinadas áreas podría traer como consecuencia una sobreexplotación de los recursos utilizados y generar conflictos entre los propios comuneros. Asimismo, los resultados presentados permiten visualizar que varias de las áreas de uso frecuente de los tsimane' se encuentran fuera del ámbito del TICH. Por lo tanto, sería conveniente que esta información fuese tomada en cuenta en las políticas o estrategias de ordenamiento de territorio y de manejo de recursos naturales en la zona.

\section{Extensión e intensidad de los usos} del territorio De acuerdo con nuestros resultados, no hay evidencia de que las comunidades dedica- 
das a las actividades comerciales hagan un uso más amplio del territorio; por el contrario, las superficies aprovechadas en actividades económicas como la agricultura comercial y la extracción de jatata para la venta parecen requerir menos área de bosque que actividades de subsistencia. Esto se explicaría porque las zonas que se utilizan para agricultura y aprovechamiento de jatata son áreas definidas. En el caso de la agricultura, por lo general se desarrolla en un radio aproximado de $2 \mathrm{~km}$ del área nucleada de la comunidad, mientras que la jatata sólo crece en lugares específicos donde encuentra condiciones edáficas y ecológicas adecuadas (Moraes y Sarmiento, 1999; Montoya, 2001). Por tanto, el uso para actividades comerciales se diferencia del uso del territorio para actividades de subsistencia extensivas, como la caza, que al ser una actividad más oportunista, se desarrolla en un territorio que puede variar mucho, pudiéndose realizar cerca de los campos de cultivo de la comunidad o hasta $20 \mathrm{~km}$ desde el centro de la comunidad. Otra actividad extensiva es la extracción de maderas para sus diferentes fines como la leña, la construcción, etc. Si bien la extracción de madera tradicionalmente ha tenido un carácter de subsistencia, actualmente también tiene un matiz comercial, especialmente para ciertas especies. De esta forma, la extracción de maderas comerciales se vuelve una actividad más selectiva y por la cual las distancias recorridas y áreas para su extracción son mayores que otras actividades, tomando en consideración que gran parte de los bosques de la zona ha estado sometida por décadas a una constante extracción de las especies más valiosas como la mara, la misma que en la década de los años 1990 ya había pasado de tener una densidad poblacional de 0,25 a 0,036 árboles por hectárea (Gullinson, 1996).

Por otro lado, aunque los datos recogidos en este estudio no permiten hacer este aná- lisis, se tendría que considerar que si bien las actividades comerciales actualmente utilizan menos espacio que la extracción de maderas y la caza, estas podrían intensificar el uso de determinada superficie, dado que, estudios previos en la zona indican que para el caso de agricultura áreas cercanas al centro poblado son deforestadas para hacer nuevos campos de cultivo. Vadez et al., (2008) determinaron que la duplicación de la superficie sembrada de arroz se asocia con un aumento del $26 \%$ al $30 \%$ en la superficie de bosque eliminado durante la próxima temporada agrícola; y en el caso de la jatata, los ciclos de corta en los jatatales son cada vez menores, con lo cual se está afectando su regeneración y reproducción (Moraes y Sarmiento, 1999). Entonces, tenemos dos cuestiones importantes en el posible efecto del proceso de integración al mercado sobre el territorio; por un lado el área total utilizada, que es el tema que analizamos en el presente artículo, y por otro lado, la intensidad de uso de esta área, aspecto que debe ser abordado en futuras investigaciones.

\section{Implicaciones para la planificación}

del territorio

Los pueblos indígenas se enfrentan a los cambios asociados con el desarrollo y al crecimiento de su población. Estos cambios incluyen modificaciones en los patrones de asentamiento, en la forma de aprovechamiento de sus recursos naturales y en sus prácticas agrícolas (Galvin, 2001). En el caso de los tsimane', si consideramos que el número de hogares en cada comunidad está asociado positivamente con en el espacio de bosque utilizado al igual que con la cercanía a carreteras, que existe también una asociación positiva entre las elasticidades del ingreso real por hogar y la deforestación (Godoy et al., 2009), y que en las últimas décadas los tsimane' han tenido una tasa de crecimiento poblacional bastante alta para 
los estándares rurales (Reyes-García, 2001), nos encontramos ante un escenario en el que la necesidad de espacio y la intensidad de aprovechamiento sobre los bosques, incluyendo su cambio de uso, será cada vez mayor. De esta forma, tener en cuenta cómo se desarrollan espacialmente las actividades de los tsimane' y cómo estas están relacionan con factores demográficos o actividades comerciales es importante en temas de planificación territorial, de gobernanza indígena y en la defensa efectiva de su territorio.

\section{Conclusión}

Este estudio analiza el área de aprovechamiento territorial de 32 comunidades tsimane'. Los resultados muestran que las comunidades tsimane' utilizan los bosques y sus recursos más allá de los límites establecidos por el gobierno boliviano, lo que indica que las TCO se otorgaron sin analizar el territorio real usado por los tsimane'. Asimismo, en el estudio se puede apreciar que en el interior del TICH existen zonas de aprovechamiento de recursos comunes a varias comunidades en las cuales se deberían establecer planes de manejo de los diferentes recursos, para asegurar la conservación del área y evitar futuros conflictos entre comunidades.
En relación con la hipótesis inicial del estudio, no se ha podido corroborar una relación clara y directa entre el vínculo de las comunidades con el mercado local (ciudad de San Borja) y un uso mayor de superficies de bosque. Es decir, al contrario de lo que habíamos propuesto, hallamos que las comunidades con una mayor distancia al mercado mostraron hacer un uso también mayor de las áreas de bosque estudiadas. Por otro lado, si se encontró que el tamaño de área utilizada está asociada en forma positiva con el número de familias de cada comunidad, con la cercanía de la comunidad a caminos o carreteras, y con el ingreso de un hogar promedio (aunque esta variable ingreso no necesariamente se vincula en forma plena con las actividades comerciales, sino también con el trabajo asalariado).

Finalmente, cabe la posibilidad de que las comunidades más lejanas al mercado usen mayores áreas de bosque pero con una intensidad menor, pudiendo conservar mejor la superficie bajo uso; mientras las comunidades cercanas al mercado hacen usos más intensivos deáreas más pequeñas afectando más el área bajo aprovechamiento. Sin embargo, esta es solo una hipótesis de trabajo que requeriría ser comprobada con datos más específicos.

\section{Agradecimientos}

Esta investigación se ha realizado dentro del marco del proyecto "Conservación del Bosque Amazónico y Territorios Indígenas: del conflicto a la colaboración. Estudio de caso en la Amazonía Boliviana" (BIOCON-06-106-07) financiado por la Fundación BBVA. Nuestro agradecimiento a la Fundación Carolina y a la Universidad Nacional Agraria La Molina (UNALM) por el financiamiento y licencias otorgadas. 


\section{REFERENCIAS BIBLIOGRÁFICAS}

Assies, W., (2006) "Land tenure legislation in a pluri-cultural and multi-ethnic society: The case of Bolivia", en Journal of Peasant Studies 33 (4), 569-611.

Apaza, L., (2002) "Estudio comparativo de la caza y uso de mamíferos en dos comunidades Tsimane'. Provincia Ballivián - Beni”. Tesis. Universidad Mayor de San Andrés. La Paz, Bolivia.

Chapin, M., Lamb, Z., \& Threlkeld, B., (2005) "Mapping Indigenous Lands", en Annual Review of Anthropoly 34, 619-638.

Chicchón, A., (1994) "El uso de la fauna en la Amazonia: Ios límites de la sostenibilidad", en Dancourt, O., Mayer, E. \& Monge, C. (eds.), El problema agrario en debate SEPIAV. Lima, Perú, pp. 669-724.

De Soto, H., (2009) “El Misterio del Capital de los Indígenas Amazónicos”. Instituto Libertad y Democracia. Lima, Perú. Documental.

De Soto, H., (2010) "La Amazonia no es Avatar. En honor a los indígenas y policías muertos en Bagua el 05 de junio del 2009". Suplemento contratado Instituto Libertad y Democracia. Lima, Perú.

Galvin, K. A., (2001) "Ecological impact of hunter-gatherers societies", en Levin, S. A. (ed.). Encyclopedia of Biodiversity, Volume 3, pp. 411-415. Academic Press.

García. A., (2007). "El Síndrome del Perro del Hortelano". Diario El Comercio.

URL: http://elcomercio.pe/edicionimpresa/ html/2007-10-/el_sindrome_del_perro_del_ hort.html: Consultado el 29 de mayo del 2010.
Godoy, R.A., Reyes-García, V., Byron, E., Leonard, W.R., \& Vadez, V., (2005) "The Effect of Market Economies on the WellBeing of Indigenous Peoples and on Their Use of Renewable Natural Resources", en Annual Review of Anthropoly 34, 121-38.

Godoy, R.A., Reyes-García, V., Vadez, V., Leonard, W.R., Tanner, S., Huanca, T., Wilkie, D., \& TAPS Bolivia Study Team., (2009) "The relation between forest clearance and household income among native Amazonians: Results from the Tsimane' Amazonian panel study, Bolivia", en Ecological Economics 68, 1864-1871.

Guèze, M., (2011) "Evaluation of tree diversity and utilization: the role of acculturation. A case study in the Bolivian Amazon". Tesis Doctoral. Universidad Autónoma de BarceIona, España.

Gujarati, D. \& Porter, D., (2010) "Econometría”. 5ta edición: Mc Graw Hill, Santa Fé de Bogotá, Colombia.

Gullison, E., Panfil, S.N., Strouse, J., \& Hubbell, S., (1996) "Ecology and management of mahogany (Swietenia macrophylla King) in the Chimanes Forest, Beni, Bolivia", en Botanical Journal of the Linnean Society 122 (1), 9-34.

Gutierrez, Y., (2005) "Uso de Avifauna por Comunidades Tsimane' y Estudio de la Comunidad: Chirisi Beni-Bolivia". Tesis. Universidad Mayor de San Andrés. La Paz, Bolivia.

Huanca, T., (1999). “Tsimane' indigenous knowledge, swidden fallow management, and conservation", Gainesville: University of Florida Press. 
Lieberman, M., (2000) "Traditional Goods and the Modern Marketplace: Preference for Commercial Substitutes and Knowledge of Traditionally-Used Plants Among the Tsimane' Indians of Lowland Bolivia". University of Florida.

Melgar, T., (2009) "Detrás del cristal con que se mira: Mujeres Chimanes, órdenes normativos e interlegalidad". Gran Consejo Chimane, Centro de Investigación y Documentación para el Desarrollo del Beni, Coordinadora de la Mujer. La Paz, Bolivia.

Montoya, P., (2001) "Guía para el manejo y aprovechamiento del palmiche o jatata: Geonomadeversa". Convenio André Bello Serie Ciencia y Tecnología 94. Bogotá, p. 46.

Moraes, M., Sarmiento, J., (1999) “La jatata (Geonoma deversa (Poit.) Kunth, Palmae) un ejemplo de producto forestal no maderable en Bolivia: uso tradicional en el Este del departamento de La Paz", en Revista de la Sociedad. Boliviana de Botánica 2, 183-196.

Organización Internacional del Trabajo, (2009) "La aplicación del Convenio Número 169 por tribunales nacionales e internacionales en América Latina: una compilación de casos". Ginebra, p. 201.

Pacheco, P., (2006) "Agricultural expansion and deforestation in lowland Bolivia: the import substitution versus the structural adjustment model", en Land Use Policy 23 (3), 205-225.

Pérez, E., (2001) "Uso de la Ictiofauna por dos comunidades Tsimane': San Antonio y Yaranda(T.I. Tsimane', Dpto. Beni). Bajo Diferente Influencia del Mercado". Tesis. Universidad Mayor de San Andrés. La Paz, Bolivia.
Redford, K.H., (1991) "The Ecologically Noble Savage", en Cultural Survival Quarterly 15 (1), 46-48.

Reyes-García, V., (2001) "Indigenous People, Ethnobotanical Knowledge, and Market Economy: A Study of the Tsimane' Amerindians, Bolivia". Gainesville: University of Florida Press.

Reyes-García, R., Godoy, R.A, Apaza, L., Byron, E., Leonard, W.R., Pérez, E., Vadez, V., \& Wilkie, D., (2002) "El valor económico del bosque para los indígenas: Estudio de caso de los Tsimane' de la Amazonía boliviana", en: Soria, C. Amazonía: Problemas del desarrollo. Universidad Nacional Mayor de San Marcos. Lima, Perú.

Reyes-García, V., Vadez, V., Aragon, J., Huanca,T., \& Jagger, P., (2010) "The uneven reach of decentralization: A case study among Tsimane' indigenous peoples in the Bolivian Amazon", en International Political Science Review 31(2), 29-43.

Reyes-García V., Ledezma, J. C., PanequeGalvez, J., Orta-Martínez, M., Guèze, M., Lobo, A., Guinard, D., Huanca, T., Luz,A. C., \& TAPS Bolivia Study Team. (2012a) "Presence and purpose of non-indigenous peoples on indigenous lands. A descriptive account from the Bolivian Lowlands", en Society and Natural Resources 25(3), 270284.

Reyes-García V., Orta-Martínez, M., Guèze, M., Luz, A. C., Paneque-Gálvez, J., Macia, M., Pino, J., Cristobal, J., \& TAPS Bolivian Study Team. (2012b) "Does participatory mapping increase conflict? A randomized experimental evaluation in the Bolivian Amazon", en Applied Geography 34, 650-658. 
Rudel, T.K., Bates, D., \& Machinguiashi, R., (2002) "Ecologically Noble Amerindians? Cattle Ranching and Cash Cropping among Shuar and Colonists in Ecuador", en Latin American Research Review 37, 144-159.

Smith, R., (2003) “Un Tapiz Tejido a Partir de las Vicisitudes de la Historia, el Lugar y la Vida Cotidiana. Imaginando los Desafíos para los Pueblos Indígenas de América Latina en el Nuevo Milenio". Oxfam America y Ford Foundation. Lima, Perú.

Sobrevila, C., (2008) "The Role of Indigenous Peoples in Biodiversity Conservation. The Natural but often Forgotten Partners. The International Bank for Reconstruction and Development". The World Bank. Washington, D.C., U.S.A.

Stocks, A., (2005) "Too much for too few: Problems of indigenous land rights in Latin America", en Annual Review of Anthropoly 34, 85-104.

Superintendencia Agraria, (2001) "Mapa de Cobertura de Uso Actual de la Tierra. Bolivia", URL:http://essm.tamu.edu/bolivia/info_ geoespacial_vector_es.htm

Consultado el 15 mayo de 2010.

Toledo, V. M., Ortiz-Espejel, B., Cortés, L., Moguel, P., \& Ordoñez, M.D,J., (2003) "The multiple use of tropical forests by indigenous peoples in Mexico: a case of adaptive management", en Conservation Ecology 7(3): 9.

Vadez, V., Reyes-García, V., Godoy, R.A., Apaza, L., Byron, E., Huanca, T., Leonard, W.R., Pérez, E., \& Wilkie, D., (2004) "Does Integration to the Market Threaten Agricultural Diversity? Panel and Cross-Sectional Data from a Horticultural-Foraging Society in the Bolivian Amazon", en Human Ecology $32,635-646$.

Vadez, V., Reyes-García, V., Huanca, T., \& Leonard, W.R., (2008) "Cash cropping, farm technologies, and deforestation: What are the connections? A model with empirical data from the Bolivian Amazon", en Human Organization 67, 384-396.

Wooldridge, J., (2010) "Introducción a la Econometría: Un Enfoque Moderno". CENGAGE Learning Editores, 4ta edición. 